Journal of Engineering and Science Research 4 (1): 47-51, 2020

e-ISSN: 2289-7127

(C) RMP Publications, 2020

DOI: $10.26666 / \mathrm{rmp}$.jesr.2020.1.8

\title{
Short Term Effect of Virtual Reality Headset on Blink Rate and Inter-Blink Interval
}

\author{
Nurul Hafizah Zaini ${ }^{1}$, Mohd Zulfaezal Che Azemin ${ }^{1}$, Mohd Hafidz Ithnin ${ }^{1}$ and \\ Mohd Izzuddin Mohd Tamrin ${ }^{2}$ \\ ${ }^{1}$ Kulliyyah of Allied Health Sciences, International Islamic University Malaysia, \\ Bandar Indera Mahkota, 25200 Kuantan, Malaysia. \\ ${ }^{2}$ Kulliyyah of ICT, International Islamic University Malaysia, Gombak, 53100 Kuala Lumpur, \\ Malaysia.
}

\begin{abstract}
Virtual reality (VR) headsets are becoming remarkably well-known nowadays, especially in gaming industry. Their ability to immerse users into virtual world makes them captivating. However, there is limited research about the impacts of this technology on our eyes and vision. This study investigated if there is any effect on blink rate (BR) and inter-blink interval (IBI) after 30 minutes of watching 3-dimensional (3D) movie on VR. Besides, this study compares between watching 3D movie on VR headset and 2D movie on laptop. Blinks were recorded over 1 minutes for 32 participants volunteered in this study before and after 30 minutes of watching 3D movie on VR headset and 2D movie on laptop. The result of BR and IBI between pre- and post-watching 3D movie on VR was not significant ( $p=>0.05$ ). The result between watching movie on laptop and VR also not significant $(p=>0.05)$. Watching 3D movie on VR for 30 minutes resulted in no effects on blink rate and inter-blink interval of the users.
\end{abstract}

Key words: Virtual reality, Blink rate, Inter-blink interval, 3-dimensional

\section{INTRODUCTION}

Virtual reality is defined as 3-dimensional environments generated by computer which allow the users to connect with alternate realities [1]. The users can immerse themselves to varying degrees in the computers artificial world which may either be a simulation of complex data or the simulation of some form of reality. VR headsets consist of two small LCD monitors, each projected at one eye. Then, $3 \mathrm{D}$ effect or stereoscopic is created which gives users the depth perception. The distance between the monitors and eyes are very close which caused researchers curious about possible adverse consequences, especially when $\mathrm{VR}$ is used for a long time. This matter is worrying since people tend to experience eye strain whenever they focus on an object for prolonged time.

This study was designed to analyze the short-term effect of VR headset towards blink rate and inter-blink interval. The act of blinking is spontaneous which aimed to wet ocular surface and maintain an intact tear film. There was a study where they claimed that the drying and irritation of ocular surface caused tear break-up and stimulate blinking [2]. Blink rate also affected by the level of attention to a certain task. For instance, the mean rate of blinking was $22 \pm 9$ per minute under relaxed conditions, $10 \pm 6$ per minute while the subjects were reading a book at table level, and $7 \pm 7$ per minute while the subjects were viewing text on a video display terminal (VDT) [3].

On the other hand, inter-blink interval refers to the time taken between blinks. It increases when people put their attention on task given especially electronic gadgets such as smartphone and VR headset. This author [4] stated that IBI was significantly reduced for dry eye patient performing a visual task as compared to normal participant.

Corresponding Author: Mohd Zulfaezal Che Azemin, Kulliyyah of Allied Health Sciences, International Islamic University Malaysia, Bandar Indera Mahkota, 25200 Kuantan, Malaysia, email: zulfaezal@iium.edu.my. 


\section{METHODS AND MATERIALS}

The materials used in this study were Samsung Gear Virtual Reality (VR) 2017 weighed 345 grams with $101^{\circ}$ field of view and Samsung Galaxy Note 8 as display incorporated into Samsung Gear VR. Samsung Galaxy Note 8 has 6.3-inch screen with Active-Matrix Organic Light-Emitting Diode (AMOLED) Quad high definition (HD) $+2960 \times 1440$ pixels display with 552ppi. Acer Aspire 4750 notebook with 14-inch display and $1366 \times 768$ pixels was used by participants to watch 2D movie on laptop. Besides, smartphone Lenovo Vibe K5 was used to record video of pre and post of participants' blinks.

Each participant $(\mathrm{n}=32)$ watched $3 \mathrm{D}$ movie on VR headset and 2D movie on laptop for 30 minutes on two different day. Participants were students of International Islamic University Malaysia (IIUM), aged between 18-25 years (14 males and 18 females), and best corrected visual acuity (BCVA) 6/9 at distance and N5 at near. They were free from any ocular trauma, chronic systemic diseases, ocular diseases, vergence and accommodation problems. All participants were explained about this study and they were given written consent form. This study was approved by KAHS Ethics Committee (KAHS 63/18).

Participants need to go through battery of assessments which are best corrected visual acuity (BCVA), dry refraction, amplitude of accommodation (AA), near point of convergence (NPC), and slit lamp bio microscopy (SLB). The BCVA were measured using Snellen Chart while AA and NPC were measured using Royal Air Force (RAF) rule. After the participants pass the preliminary examination, their blinks were recorded for 1 minute by using smartphone Lenovo Vibe K5. The subjects were not told that their blinks were being analysed to avoid forced blinking and other changes in spontaneous blink patterns [4]. The smartphone was positioned on tripod and positioned towards the eyes so that movement of eyelid can be recorded.

Participants sat on a comfortable chair under normal office illumination with air conditioner switched off and watched 3D movie with 1080p resolution entitled 'How to Train Your Dragon 2' on Samsung Gear Virtual Reality for 30 minutes. A slight cooling generated by atmospheric air turbulence will trigger tearing which may affect the result of this study [5]. For the next visit, they watched the same movie in 2D for 30 minutes on laptop. The distance between participants and laptop was 1 metre. The short distances between the eye and the display during headmounted display (HMD) viewing of VR and high immersion experience are factors that cause a decrease in the eye blink rate [6]. Participants' blinks were videotaped after 30 minutes of watching the video. The videos were manually processed.

Statistical analysis was conducted using IBM SPSS (Version 24.0, SPSS Inc., Chicago, Illinois, USA). Paired t-test was used for parametric test while Wilcoxon signed-rank test was used for data analysis of non-parametric test. A value of $p<0.05$ was considered significant.

\section{RESULTS}

Thirty-two participants including 14 males and 18 females aged between 20-25 years old were selected in the study. None of the parameters revealed statistically significant changes in blink rate and inter-blink interval after 30 minutes of watching 3D video on VR headset (Table 1). Meanwhile, blink rate and inter-blink interval between post-watching movie on VR and laptop also give no significant differences $(p>0.05)$ result (Table 2).

\section{DISCUSSION}

Blink is vital to health of ocular surface and increases an individual's quality of life. Hence, any disturbances in blink lead to breakdown of tear film stability, visual function and optical clarity [7]. On average, human blinks approximately every 4 seconds after opening eyes under relaxed condition [8]. Any modifications in the blinking pattern might influence tear distribution and drainage, cause changes on tear volume and other parameters of tear systems [9].

Inter-blink interval revealed a meaningful amount of information for a given sample of blinks, whereby blink rate is a single measurement while inter-blink interval series provides several endpoints describing a participant's blink frequency to have better 
discrimination between normal and dry eye subjects

[4].

Table 1 Comparison of blink rate and inter-blink interval between pre and post watching 3D movie on VR.

\begin{tabular}{llllll}
\hline Parameters Studied & Pre & Post & Z-value & $t($ df $)$ & $p$-value
\end{tabular}

\begin{tabular}{|c|c|c|c|c|}
\hline BR (blink/min) * & $20.19 \pm 12.75$ & $20.13 \pm 10.70$ & $1.015(31)$ & 0.972 \\
\hline
\end{tabular}

Blink rate (BR); Inter-blink interval (IBI)

*Data displayed in mean \pm standard deviation; $p$-value analysed using paired sample $t$-test.

+ Data displayed in median \pm interquartile range; $p$-value analysed using Wilcoxon Signed-rank.

Table 2 Comparison between blink rate and inter-blink interval post watching movie on VR and on laptop.

\begin{tabular}{llllll}
\hline Parameters Studied & VR & Laptop & Z-value & $t(\mathrm{df})$ & $p$-value
\end{tabular}

\begin{tabular}{lcccc}
\hline BR (blink/min) * & $20.13 \pm 10.70$ & $18.78 \pm 12.10$ & $0.360(31)$ & 0.318 \\
& & & & \\
IBI (s) + & $3.08 \pm 3.05$ & $3.55 \pm 3.35$ & -0.916 & 0.360
\end{tabular}

Blink rate (BR); Inter-blink interval (IBI)

*Data displayed in mean \pm standard deviation; $p$-value analysed using paired sample $t$-test.

+ Data displayed in median \pm interquartile range; $p$-value analysed using Wilcoxon Signed-rank.

In this study, the blink rate for watching movies on VR ranged from 3 to 58 blinks/minute while the mean of inter-blink interval ranges from 0.80 to 18.04 . Based on the data above, there were no statistically significant changes on BR and IBI associated with 30 minutes use of virtual reality headset. Also, comparison between watching on laptop and VR resulted in no significant difference after 30 minutes of watching videos on BR and IBI. Thus, VR is safe to be used up to 30 minutes. However, this paper [6] suggested having temporary cessation of HMD usage if there is feeling of dryness on eyes to rest the eyes and parental guidance are needed for children. This paper [10] claimed that short term use of HMD reduces TBUT contributed to dry eye symptoms.

The investigation by this paper [4] showed reduced IBI in dry eye participants when performing visual task compared to normal participants. Same goes to this study [2] whereby there were reduction in BR and IBI of participants while watching a televised documentary. However, their IBI in both studies were recorded during performing visual task while current study was recorded before and after performing visual task. In addition, both studies only using computer while current study compared between laptop and VR. 
On the other hand, the procedure conducted in this study [11] almost like current study since blink interval was recorded before and right after visual task on computer which gave result of decreased maximal blinking interval, ocular protection index and tear break-up time. However, the task was conducted for an hour using computer only, compared to current study which was 30 minutes on VR and laptop each.

There was a study conducted by [10] which investigated short term effect of HMD and computer usage on functional changes in binocular changes in binocular vision, accommodation and resolution after 30 minutes usage which did not include blink rate and inter-blink interval. None of the parameters from the study displayed statistically significant result. Also, there was no changes in any of parameters were statistically different from computer.

The insignificant result in this current study may be due to lack of observation on participants' eye movement during watching movie especially when using VR since their eyes were completely covered with the VR headset. The latest study by [12] proposed a real-time eyeblink detector to detect blinks when using VR headset which may be helpful for future studies to monitor BR and IBI while wearing VR headset.

Other than that, participants should be grouped into dry eye patient and healthy participants to have more reliable results. Hence, tear break-up time (TBUT) test should be included in preliminary test. This paper [13] stated that the blink rate and maximal blink interval were significantly different between dry eye patients and healthy participants, with the values of dry eye patient approaching healthy participants after instillation of false tears.

\section{CONCLUSION}

In conclusion, VR gives no significant effects on blink rate and inter-blink interval. Hence, VR is suggested safe to be used up to 30 minutes.

\section{ACKNOWLEDGEMENT}

This work is supported by the International Islamic University Malaysia (IIUM), under the Research Initiative Grant (P-RIGS18-035-0035).

\section{REFERENCES}

[1] Dam, A.V., Forsberg, A., Laidlaw, D., Laviola, J. and Simpson, R. 2000. Immersive VR for scientific visualization: A progress report. IEEE Computer Graphics and Applications, 20, 6, 26-52, DOI:10.1109/38.888006.

[2] Acosta, M., Gallar, J. and Belmonte, C. 1999. The Influence of Eye Solutions on Blinking and Ocular Comfort at Rest and During Work at Video Display Terminals. Experimental Eye Research, 68, 6, 663669, DOI:10.1006/exer.1998.0656.

[3] Tsubota, K. and Nakamori, K. 1993. Dry Eyes and Video Display Terminals. New England Journal of Medicine, 328, $\quad 8, \quad 584-584$, DOI:10.1056/nejm199302253280817.

[4] Johnston, P., Rodriguez, J., Lane, K., Ousler and Abelson, M. 2013. The interblink interval in normal and dry eye subjects. Clinical Ophthalmology, 7, 253-259, DOI:10.2147/opth.s39104.

[5] Hirata, H., Dallacasagrande, V., Mizerska, K., Ivakhnitskaia, E. and Rosenblatt, M.I. 2018. Ambient Air Currents Activate Corneal Nerves During Ocular Desiccation in Rats: Simultaneous Recordings of Neural Activity and Corneal Temperature. Investigative Opthalmology \& Visual Science, 59, 10, 4031-4043, DOI: 10.1167/iovs.1824604.

[6] Kim, J., Kumar, Y.S., Yoo, J. and Kwon, S. 2018. Change of Blink Rate in Viewing Virtual Reality with HMD. Symmetry, 10, 9, 400, DOI: 10.3390/sym10090400.

[7] Rodriguez, J.D., Lane, K.J., Ousler, G.W., Angjeli, E., Smith, L.M. and Abelson, M.B. 2017. Blink: Characteristics, Controls, and Relation to Dry Eyes. Current Eye Research, 43, 1, 52-66, DOI:10.1080/02713683.2017.1381270.

[8] Tsubota, K. (1998). Tear dynamics and dry eye. Progress in Retinal and Eye Research, 17(4), 565-596. doi: 10.1016/s1350-9462(98)00004-4

[9] Palakuru, J.R., Wang, J. and Aquavella, J.V. 2007. Effect of Blinking on Tear Dynamics. Investigative Opthalmology \& Visual Science, 48, 7, 3032, DOI: 10.1167/iovs.06-1507.

[10] Peli, E. 1998. The visual effects of head-mounted display (HMD) are not distinguishable from those of desk-top computer display. Vision Research, 38, 13, 2053-2066, DOI:10.1016/s0042-6989(97)00397-0.

[11] Suh, Y. W., Kim, K. H., Kang, S. Y., Kim, S. W., Oh, J. R., Kim, H. M., \& Song, J. S. (2010). The Objective Methods to Evaluate Ocular Fatigue Associated With Computer Work. Journal of the Korean Ophthalmological Society, 51(10), 1327. doi: $10.3341 /$ jkos.2010.51.10.1327

[12] Alsaeedi, N. and Wloka, D. 2019. Real-Time Eyeblink Detector and Eye State Classifier for 
Mohd Zulfaezal Che Azemin et. al./ Journal of Engineering and Science Research, 4(1) 2020, Pages:47-51

Virtual Reality (VR) Headsets (Head-Mounted Displays, HMDs). Sensors, 19, 5, 1121, DOI: $10.3390 / \mathrm{s} 19051121$.

[13] Nakamori, K., Odawara, M., Nakajima, T., Mizutani, T. and Tsubota, K. 1997. Blinking Is Controlled Primarily by Ocular Surface Conditions. American Journal of Ophthalmology, 124, 1, 24-30, DOI: $10.1016 / \mathrm{s} 0002-9394(14) 71639-3$. 